\title{
Ambulatory colectomy: fast-track management pushed to its limits?
}

\author{
J.-L. Faucheron ${ }^{1,2} \cdot$ B. Trilling ${ }^{1,2} \cdot$ P.-Y. Sage ${ }^{1}$
}

Received: 5 October 2018 / Accepted: 19 October 2018 / Published online: 9 November 2018

(c) Springer Nature Switzerland AG 2018

Enhanced recovery after surgery provides patients with optimal means to counteract or minimize the deleterious effects of surgery [1-3]. This concept can be adapted to suit colorectal procedures and comes in the form of a clinical pathway covering the pre-, per- and postoperative periods. Enhanced recovery after colorectal surgery includes preoperative, intraoperative, and postoperative measures and consists of a multidisciplinary approach involving surgeons, anesthesiologists, nurses, dieticians and physiotherapists. A French expert panel assessed the impact of parameters included in the fast-track programs on six foreseeable consequences of colorectal surgery; these were surgical stress, postoperative ileus, fluid and electrolyte imbalances, decreased postoperative mobility, sleep disorders, and postoperative complications. The experts validated the value of each parameter in terms of efficacy criteria for success of fast-track programs. After a review of the literature, 19 parameters were identified as potentially impacting at least one of the foreseeable consequences of colorectal surgery, namely the rate of postoperative complications and the duration of hospital stay. After synthesis of the work of experts on the 19 parameters using a well-defined methodology, the organizing committee reached 35 formal recommendations that were published as guidelines [4]. In a prospective randomized trial, Vlug et al. demonstrated that laparoscopy in combination with fast-track multimodal management was the best strategy over laparoscopy in a standard care or open surgery in either fast-track or standard care [5]. This particularly applies to colectomy or proctectomy for cancer [6].

Consensus was reached among anesthesiologists, nutritionists, physiotherapists, and surgeons on a number of recommendations that should be applied in current

J.-L. Faucheron

JLFaucheron@chu-grenoble.fr

1 Colorectal Unit, Department of Surgery, Michallon

University Hospital, Grenoble, CS 10217 ,

38043 Grenoble Cedex, France

2 University Grenoble Alpes, Grenoble, France rehabilitation programs in colorectal surgery [7] such as: preoperative immunonutrition; preoperative intake of carbohydrates until $2 \mathrm{~h}$ before surgery; no bowel preparation for colon surgery and bowel preparation for rectal surgery; laparoscopic approach; antibiotic prophylaxis; prevention of hypothermia; systematic intraoperative medication to prevent nausea and vomiting; morphine-sparing analgesia techniques; optimization of intraoperative volume control; no gastric tube; no abdominal drainage; short-term bladder catheterization; resumption of oral feeding within $24 \mathrm{~h}$; getting the patient out of bed and walking on the first day. Patient information has also been considered as an important point for a fast-track program.

Several studies showed that an enhanced recovery protocol for colorectal surgery reduces postoperative morbidity, and mortality [8]. The development of such an enhanced recovery program also allowed gradual shortening of hospital stay for colorectal surgery patients [9].

Applying the concept of fast-track surgery, we formalized a clinical pathway protocol for laparoscopic colectomy performed in an ambulatory setting according to the International Association for Ambulatory Surgery definition that is discharge on the same working day without overnight stay [10]. The short-term outcomes of ambulatory colectomy for the 157 consecutive patients who followed this program at two institutions has now been published [11]. In our study, the admission rate was $7.0 \%$, the unscheduled consultation rate was $20.5 \%$, the readmission rate was $6.1 \%$, and the reoperation rate was $3.8 \%$. The overall 30 -day morbidity rate was $24.8 \%$ and the mortality rate was $0 \%$. These results are similar to those published in the current literature concerning colorectal resection imbedded in enhanced recovery programs [9].

It seems that the project of enhanced recovery programs after colorectal surgery has reached the last phase of its development. The experience of the entire team with a fasttrack protocol, the assistance from the institutional support system and the involvement of primary care physicians are necessary prerequisites for ambulatory colectomy. The careful post-discharge surveillance, particularly with the help of 
nurses well-trained in enhanced recovery, allows reviewing patients for suspicion of medical or surgical complications. Another useful tool for ambulatory colectomy success is the use of post-discharge text messages (four simple questions with regard to pain, bowel movements, temperature and malaise) that are sent to the patients regularly, for example, on days 1, 3 and 5. In case of an abnormal response or lack of response, an automatic alert is sent to the attending physician via internet and the patient is contacted immediately. Surveillance using text messages has been validated for minor operations in ambulatory surgery and for operations in enhanced recovery programs [12].

Lastly, motivated patients in good general condition with no or few comorbidities, scheduled colon resection without foreseeable difficulties, and a thorough understanding of the ambulatory process and surveillance are crucial points for true ambulatory colectomy.

In conclusion, the feasibility, safety, and reproducibility of outpatient colectomy for selected patients has now been demonstrated [11]. Very short hospital stays after colectomy require establishment of a specific at-home monitoring system to avoid any delay in the diagnosis and management of life-threatening complications. In experienced centers, nearly $30 \%$ of patients scheduled for elective colectomy can be managed in an ambulatory setting [11]. This strategy can be considered as the ultimate development of enhanced recovery programs.

\section{Compliance with ethical standards}

Conflict of interest The authors declare that they have no conflict of interest.

Ethical approval For this type of study ethical approval is not required.

Informed consent For this type of study formal consent is not required.
2. Lohsiriwat V (2014) The influence of preoperative nutritional status on the outcomes of an enhanced recovery after surgery (ERAS) program for colorectal cancer surgery. Tech Coloproctol 18:1075-1080

3. Barr J, Boulind C, Foster JD et al (2015) Impact of analgesic modality on stress response following laparoscopic colorectal surgery: a post-hoc analysis of a randomised controlled trial. Tech Coloproctol 19:231-239

4. Alfonsi P, Slim K, Chauvin M, Mariani P, Faucheron JL, Fletcher D (2014) French guidelines for enhanced recovery after elective colorectal surgery; Société française d'anesthésie et réanimation (SFAR); Société française de chirurgie digestive (SFCD). J Visc Surg 151:65-79

5. Vlug MS, Wind J, Hollmann MW et al (2011) Laparoscopy in combination with fast track multimodal management is the best perioperative strategy in patients undergoing colonic surgery. A randomized clinical trial (LAFA-study). Ann Surg 254:868-875

6. Faucheron JL, Trilling B (2015) Laparoscopy in combination with fast track management is the best surgical strategy in patients undergoing colorectal resection for cancer. Tech Coloproctol 19:379-380

7. Trilling B, Sage PY, Faucheron JL (2018) What is fast track multimodal management of colorectal cancer surgery in real life? Tech Coloproctol 22:401-402

8. Lassen K, Soop M, Nygren J et al (2009) Consensus review of optimal perioperative care in colorectal surgery-Enhanced Recovery After Surgery (ERAS) Group recommendations. Arch Surg 144:961-969

9. Pearsall EA, Meghji Z, Pitzul KB et al (2015) A qualitative study to understand the barriers and enablers in implementing an enhanced recovery after surgery program. Ann Surg 261:92-96

10. Vinson-Bonnet B, Juguet F, Faucheron JL, the French National Coloproctology Society (2015) Ambulatory proctologic surgery: recommendations of the French National Coloproctology Society (SNFCP). J Visc Surg 152:369-372

11. Gignoux B, Gosgnach M, Lanz T, Vulliez A, Blanchet MC, Frering V, Faucheron JL, Chasserant P (2018) Short-term outcomes of ambulatory colectomy for 157 consecutive patients. Ann Surg. https://doi.org/10.1097/SLA.0000000000002800 (Epub ahead of print)

12. Carrier G, Cotte E, Beyer-Berjot L, Faucheron JL, Joris J, Slim K, Groupe Francophone de Réhabilitation Amélioree après Chirurgie GRACE (2016) Post-discharge follow-up using text messaging within an enhanced recovery program after colorectal surgery. J Visc Surg 153:249-252

\section{References}

1. Kehlet H, Wilmore DW (2008) Evidence-based surgical care and the evolution of fast-track surgery. Ann Surg 248:189-198 